\title{
Design and Development of Circuit Board for Serial Interface Technology
}

\author{
Maosheng $\mathrm{Wu}$ \\ College of Computer Science and Software \\ Zhaoqing University \\ Zhaoqing, China \\ 397221926@qq.com
}

\author{
Huizhang Zhang \\ College of Computer Science and Software \\ Zhaoqing University \\ Zhaoqing, China \\ 651193157@qq.com
}

\begin{abstract}
In order to help students learn and master SCM serial interface technology, one self-teaching circuit board on serial interface technology has been designed. This paper mainly discusses the design method of the circuit board. First of all, the on-system programming module, $I^{2} \mathrm{C}$ module, SPI module, 1-Wire bus module, serial keyboard and digital tube and LCD display interface module are introduced in detail; then the design method of on-system programming software is discussed. With this circuit board, students can easily master all kinds of serial interface technology usually used in the development of single chip microcomputer. It has been used by many colleges and universities and SCM technology training, the good teaching effect has been achieved.
\end{abstract}

Keywords-Microcontroller; serial interface; Experimental circuit board; In-System Programming

\section{INTRODUCTION}

Owing to its small volume, light weight, strong functions, low power consumption, and easily distributed control and so on, microcontroller (sometimes also called "MCU" or "SCM") has been widely used since its birth[1]. Therefore, At present, the course of the principle and application of the microcontroller is offered for students on more than 10 disciplines. This is a highly practical course, The key to good study is to do well experiment[2]. However, during the current SCM technology teaching, there is a commonly problem of experimental hours shortage, which seriously affects the teaching effect. On the other hand, Because the serial interface technology uses fewer data lines for data transmission, Using the serial interface chip design circuit can not only reduce the size of the PCB board, save the decoding circuit, reduce the cost, but also reduce system power consumption and improve reliability, The serial interface technology has become an indispensable technology in SCM development. Therefore, self-study circuit board on serial interface technology has been designed. With this circuit board, students can easily master all kinds of serial interface technologies commonly used in SCM development, such as $\mathrm{I}^{2} \mathrm{C}$, SPI, 1-Wire and USB.

\section{THE FUNCTION OF CIRCUIT BOARD}

The serial interface technology self-study circuit board (SITCB) is mainly used for learning or demonstration of $\mathrm{I}^{2} \mathrm{C}$, SPI, 1-wire, USB and other serial bus technology. In addition to the function is very rich, it can also be connected to the computer and run the debugger online. Both the source code of assembly language and the source program written by Keil C51 can be executed step by step on the computer. At the same time, the value of the MCU internal registers and memory and each instruction actual operation results on the experimental board can be seen. In other words, the experimental board has the function of the hardware emulator.

\section{SYSTEM IMPLEMENTATION}

\section{A. Hardware circuit design}

The SITCB hardware includes in-system programming module to the AT89S51 MCU, I ${ }^{2} \mathrm{C}$ interface module, SPI interface module, 1-Wire bus module, serial keyboard and digital tube interface module and serial 12864LCD interface module etc.. The shape of the SITCB is shown in Fig. 1.

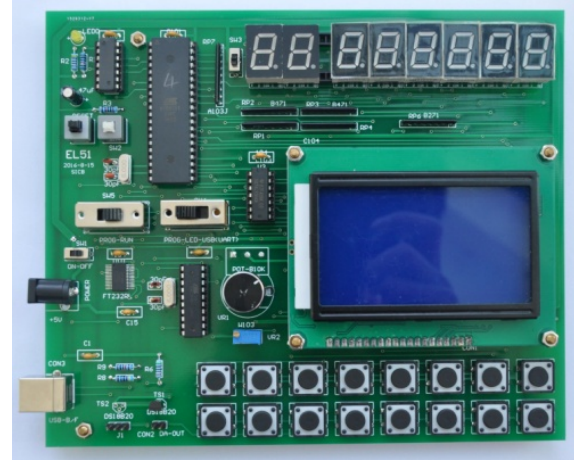

Fig. 1. The serial interface technology circuit board

\section{1) Design of In-system programming module}

In the experiment board design, the first problem to be solved is how to program the microcontroller. The In-system programming module is one of the most important modules in the experiment board. The AT89S51 is a low-power, high-performance CMOS 8-bit microcontroller with $4 \mathrm{~K}$ bytes of in-system programmable flash memory. Its instruction and pin distribution is fully compatible with the standard $80 \mathrm{C} 51^{[3]}$. When the RESET pin is high level, it can be In-system programmed by means of P1.7 (clock signal SCK), P1.6 (MISO, output) and P1.5 (MOSI, input). The programming module hardware is shown in Fig. 2. 


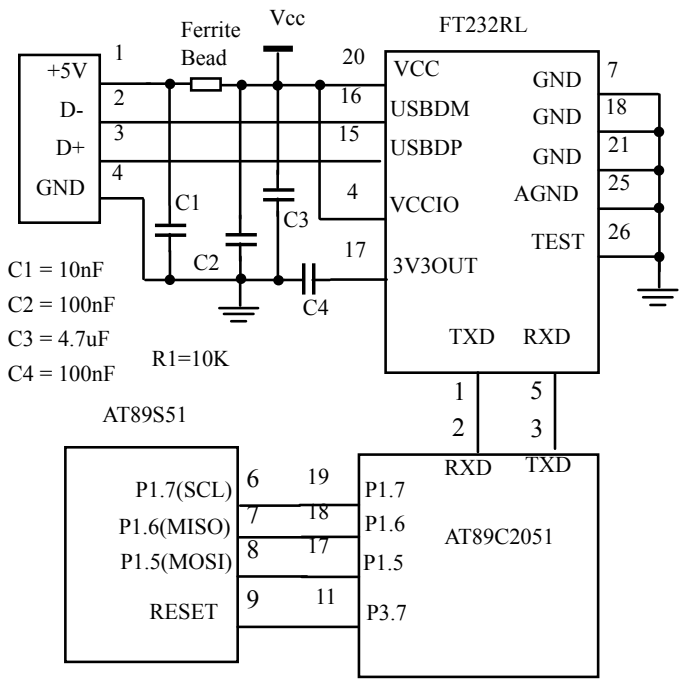

Fig. 2. In-System Programming Unit Circuit

In Fig. 2, the programming software first transfers the program code to AT89C2051 by computer USB port, each block of 32 bytes, when data transmission correct, according to the serial programming sequence of AT89S51, AT89C2015 programmed to At89S51 and checked. At last, AT89C2051 sends an answering message to the host computer to indicate whether the programming is success. In order to reduce the difficulty of writing programming software, the USB to UART chip FT232RL was used in Fig. 2. The FT232RL, designed by Future Technology Devices International Ltd. (referred to FTDI) in the United Kingdom, is a USB to serial UART interface with the following advanced features: single chip USB to asynchronous serial data transfer interface, entire USB protocol handled on the chip, no USB specific firmware programming required. Fully integrated 1024 bit EEPROM storing device descriptors, USB termination resistors, power-on-reset circuit and clock generation with no external crystal required etc. Because FTDI provides drivers, it is very easy to use the chip. Before operating AT89S51, 4 byte serial programming enable instruction was executed firstly. Then, executing chip erase command can erase flash memory array, and the content after the chip erased is FFH. Performing byte reading/ writing instruction or page reading/ writing instructions can complete read and write operations on the chip. When data is transmitted, the most significant bit (MSB)of data is sent first. When in-system programming, after the falling edge of the SCK, the AT89C2051 writes 1 bit to the P1.5 of the AT89S51. When reading data, the falling edge of the previous SCK has output 1 bit data to the P1.6 pin of the AT89S51, and after the SCK becomes high, read the P1.6 pin of the AT89S51. The assembly language program of reading and writing a byte is as follows.

SCK BIT P1.7; clock

MOSI BIT P1.5; programming(writing)

MISO BIT P1.6; verification (reading)

MEM EQU $30 \mathrm{H}$; data buffer

; The subroutine reading one byte

INBYTE:

MOV R3, \#8

INB: CLR SCK
NOP

SETB SCK ; setting SCK as high level

NOP

MOV C, MISO

RLC A

DJNZ R3, INB

MOV MEM, A

CLR SCK

RET

; The subroutine of writing one byte

OUTBYTE:

MOV A, MEM

OUTB:

MOV R3, \#8

CLR SCK ; setting SCK as low level

NOP

RLC A

MOV MOSI, C

NOP

SETB SCK

DJNZ R3, OUTB

LCALL DLT500us ; delay 500 $\mu$ s

CLR SCK

RET

2) Design of I2C technology module

$\mathrm{I}^{2} \mathrm{C}$-bus is a simple bidirectional 2-wire bus developed by Philips Semiconductors (now NXP semiconductors), Data transmission is accomplished by two lines SDA (Serial, Data) and clock line SCL (Serial, Clock), Using software addressing, without hardware decoding circuit, all the $\mathrm{I}^{2} \mathrm{C}$ interface devices are connected to microcontroller through the two lines, so that the system can be easily extended. Since the output of $\mathrm{I}^{2} \mathrm{C}$ devices usually adopts open drain structure, external pull-up resistor is needed. Due to the advantages of $\mathrm{I}^{2} \mathrm{C}$ bus, at present, there are many of the world's other semiconductor manufacturers that have developed hundreds of different kinds of functional chip of using the technology of $\mathrm{I}^{2} \mathrm{C}$ bus, , completing data storage, $\mathrm{A} / \mathrm{D}, \mathrm{D} / \mathrm{A}, \mathrm{LED} / \mathrm{LCD}$ display driver, calendar clock. $\mathrm{I}^{2} \mathrm{C}$ bus has also become one of the standard interfaces of ARM microcontroller. In order to facilitate the learning of $\mathrm{I}^{2} \mathrm{C}$ bus interface technology, the serial interface board includes two $\mathrm{I}^{2} \mathrm{C}$ chips; they are EEPROM memory chip $\mathrm{AT} 24 \mathrm{C} 02$ and $\mathrm{A} / \mathrm{D}$ and $\mathrm{D} / \mathrm{A}$ conversion chip PCF8591. AT24C02 is the E2PROM chip designed by the world's most famous semiconductor manufacturers Atmel, using $\mathrm{I}^{2} \mathrm{C}$ interface, providing 256 bytes serial electrically erasable and programmable read-only memory (EEPROM), working voltage: $2.5 \mathrm{~V} \sim 5.5 \mathrm{~V}$, with Hardware Data Protection. In the development of single chip microcomputer application system, it is often used to save various system parameters and important data. The interface between the AT24C02 and the microcontroller is shown in Fig. 3.

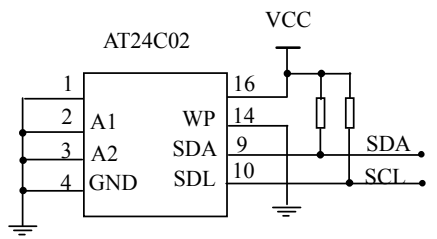

Fig. 3. Interface circuit between the AT24C02 and MCU 
The PCF8591 is a single-chip, single-supply low-power 8-bit CMOS data acquisition device with four analog inputs, one analog output and a serial $\mathrm{I}^{2} \mathrm{C}$-bus interface. $\mathrm{I}^{2} \mathrm{C}$ address was selected by address pins A0, A1 and A2, 4 analog inputs can be configured as single ended or differential inputs. The interface of the PCF8591 with the microcontroller is shown in Fig. 4.

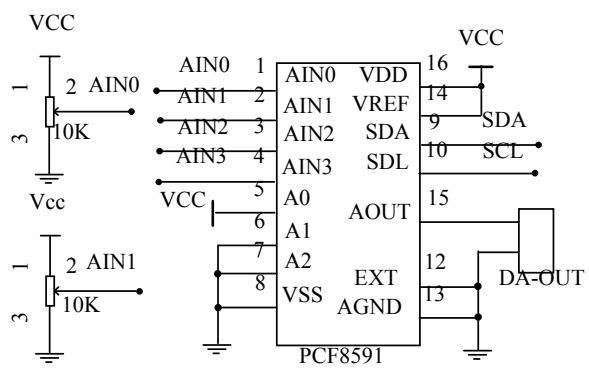

Fig. 4. Interface circuit between the PCF8591 and MCU

A HIGH to LOW transition on the SDA line while SCL is HIGH defines a START condition. A LOW to HIGH transition on the SDA line while SCL is HIGH defines a STOP condition. When data is transmitted by the $\mathrm{I}^{2} \mathrm{C}$ bus, all transactions begin with a START (S) and can be terminated by a STOP $(\mathrm{P})$, each transmits 1 bytes of data, the receiver needs to send an acknowledge, When transmitting data, the most significant bit of the byte is transmitted first, When writing data, the host computer moves 1 bit to the SDA at the falling edge of the clock signal. The rising edge of the clock signal writes the bit to the slave computer; when reading data, the falling edge of the previous clock signal moves out 1 bit data from the slave, and the host reads the data after the clock signal is raised.

\section{3) Design of SPI technology module}

SPI bus (Serial Peripheral Interface Bus) is a full duplex serial data transmission standard designed by Motorola company, it completes data transmission through the 4 signal lines SCLK (Serial Clock), MOSI (Master Output, Slave Input), MISO (Master Input, Slave Output) and SS (Slave Select). SPI bus has also become one of the standard interfaces of MCU, which is widely used in intelligent instrument and measurement and control system which needs low speed and saves a small amount of parameters. In order to learn the SPI interface technology, the serial interface board includes two chips of SPI interface, X5045 and calendar clock chip DS1302. X5045 includes the power on reset, power monitoring, chip watchdog circuit and EEPROM, designed by the American XICOR company. Using SPI interface, the main features are as follows: The watchdog timer overflow time can be programmed to 1.4 seconds, 600 milliseconds and 200 milliseconds, respectively; When the supply voltage is below the set limit, it outputs RESET signal; Containing 512 bytes of EEPROM, erasable 106 times, data is stored for 100 years. The data in EEPROM can be protected by both hardware or software methods, at most 16 bytes of data at can be written each time, the internal timing write cycle is about $5 \mathrm{~ms}$; it is one of the commonly used components of SCM development. The interface circuit of X5045 and MCU are shown in Fig. 5.

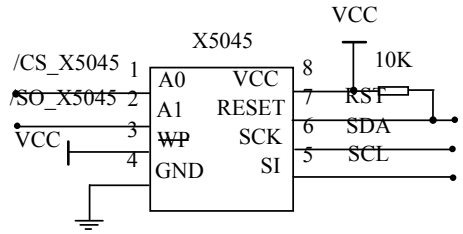

Fig. 5. Interface circuit between the X5045 and MCU

In order to access to X5045, /CS must be low. When transmitting data, the most significant bit of the byte is transmitted first. During the SCK high level, the data on the SI and SO lines is valid data. During the SCK low level, data on the SI and SO lines changes. When writing data to the X5045, the host sends data to the SI pin of the X5045 after the falling edge of the SCK, and the rising edge of the SCK writes the data into the X5045.

DS1302 is a calendar clock chip, including a real-time calendar clock and 31 bytes of SRAM, transmitting data by three lines (CE, I/O, SCLK), providing seconds, minute, week, day, month and year information; including 12 or 24 hours mode, when 12 hour mode with AM and PM flag, a byte or more than one byte of data can been read or written each time. The interface circuit of DS1302 with MCU is shown in Fig. 6.

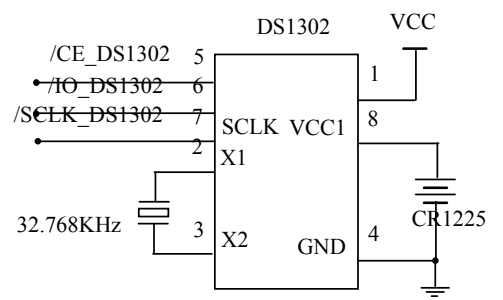

Fig. 6. Interface circuit between the X5045 and MCU

The DS1302 operation sequence is as follows: All data transfers starts from CE to high level. CE has two functions. First, open the control logic to allow access to the address or command in the shift register. Second providing a way to determine whether a single byte transmission or multi byte send data. When entering data, the data must be valid before the rising edge of SCLK; when outputting data, the data is output after the falling edge of SCLK. If the CE becomes low, all data transfers are ended, and the I/O becomes high impedance

\section{4) Design of 1-Wire bus technology module}

1-Wire Bus is a serial communication bus developed by DALLAS Semiconductor Corporation of the United States. Compared with $\mathrm{I}^{2} \mathrm{C}$ and SPI, the advantage of 1 -Wire bus is that it has longer communication distance and less data transmission line. The disadvantage is that the speed is slow. In order to study single bus technology, the digital temperature sensor DS18B20 based on 1-Wire bus is adopted. DS18B20 is a digital temperature sensor designed by the DALLAS Semiconductor Corporation of the United States. It converts the temperature directly to the digital temperature. The measuring temperature range is $-55^{\circ} \mathrm{C} \sim+125^{\circ} \mathrm{C} ; 1$-Wire bus interface. In the range of $-10^{\circ} \mathrm{C} \sim 85^{\circ} \mathrm{C}$, the measuring accuracy is up to $+0.5^{\circ} \mathrm{C}$. Temperature conversion resolution can be programmed separately for $9,10,11$ and 12 bit. The maximum 
conversion time is $750 \mathrm{~ms}$, easy to use. It is particularly suitable for a wide range of remote temperature measurement, control systems and various consumer products. The interface circuit of DS18B20 and MCU is shown in the following Fig. 7.

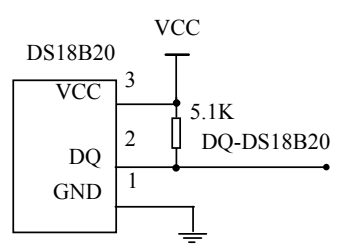

Fig. 7. The Interface circuit of the DS18B20

Operating DS18B20 must follow the following order:

The first step: executing the start sequence;

The second step: the host sends the ROM command, then followed by data exchanged

The third step: the host sends the function command, then followed by data exchanged

5) Design of serial keyboard and digital tube interface module

CH451 is a serial interface chip with digital tube display driver and keyboard scanning control and microprocessor monitoring. Compared with the Intel 8279, it has the advantages of small size, large current drive, simple peripheral interface circuit, built-in power on reset circuit and clock oscillation circuit and watch-dog circuit, external crystal or oscillator is not required, High speed 4 wire serial interface, supports multi chip cascade, clock speed is up to $10 \mathrm{MHz}$. The keyboard and digital interface circuits of the $\mathrm{CH} 451$ are shown in Fig. 8.

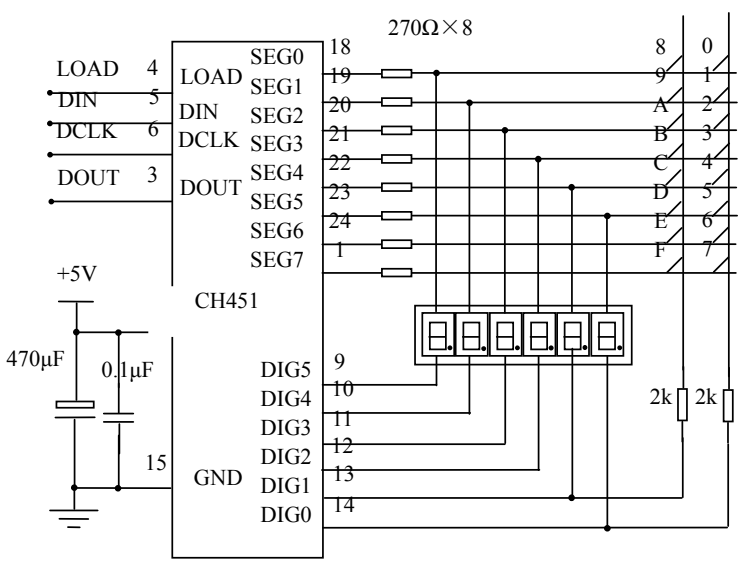

Fig. 8. The keyboard and digital interface circuits by $\mathrm{CH} 451$

6) The design of serial LCD12864 interface module

LCD is one of the most commonly used peripherals MCU application system. Compared with LED, it can display more information, such as Chinese characters, graphics and so on. In order to study the serial LCD interface and programming method, serial interface circuit board extends a liquid crystal display OCMJ4X8C. OCMJ4X8C is a $128 * 64$ dot matrix Chinese LCD module. It can display letters, numbers, Chinese characters and graphics, Built in 2M- bit Chinese font ROM (CGROM), providing 8192 Chinese character dot matrix information (16x16 dot matrix), 16K- bit half width font ROM
(HCGROM), 126 letters, numbers and other symbols dot matrix information (16x8 dot matrix). There are a lot of function instructions to control the display screen. It communicates with MCU through 3 signal line.

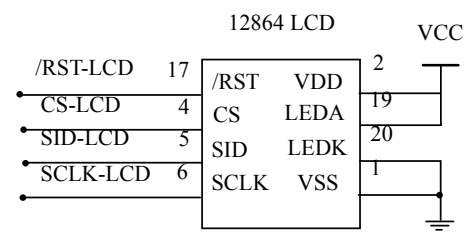

Fig. 9. the Interface circuit of serial LCD12864

CS is chip select signal; SID is serial input data; SCLK is clock signal; /RST is reset signal. When MCU sends 1 bits of information, the CS is first high, and when the clock signal SCLK is low, the host sends the data to the SID line, and the rising edge of the SCLK sends the data into the LCD module. In order to control the display of LCD, MCU also sends various instructions to the LCD module. The OCMJ4X8C LCD module has two instruction sets called the basic instruction set and the extended instruction set respectively.

\section{B. The developing software in-system programming}

SCM In-system programming software is developed in Visual Studio 2010 environment; it can complete blank check, erasing and In-system programming operation of the microcontroller. The software running interface is shown in Fig. 10.

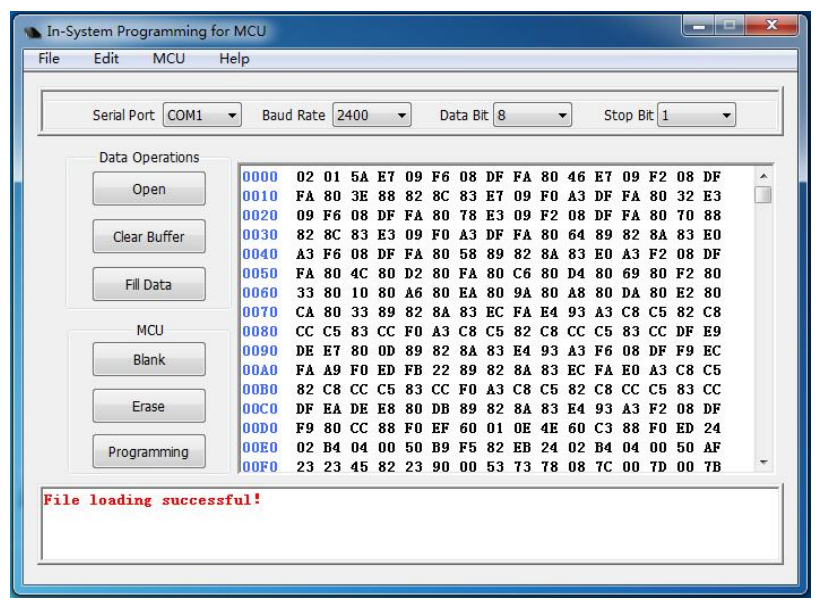

Fig. 10. In-system programming running interface

\section{1) The file operations}

The SCM code file is a text file that uses Intel HEX file format. It consists of lots of records, each row is called a record that begins with "colon" and ends by "0DH" and "0AH", each record consists of five fields ${ }^{[4]}$. The format is as follows.

: LL AAAA TT DD ... DD CC 0D0A

":" is the beginning of the record, its ASCII code is $3 \mathrm{AH}$; "LL" is the code byte numbers the record containing;

"AAAA" is code start address in record; 
"TT" indicates record type, 00 said data records; 01 said end of file;

"DD" is code data;

"CC" is record checksum.

For example, a code file displays as follows.

: $0200000080047 \mathrm{~A}$

: $1000030002001 B 758160 C 292 D 2 A F A 2934004 D 288 D 2$

: $100013008002 C 288 D 2 A 880 F E 7805 B 2927530057 F 2 F$

: $\odot$ FO०2300647E7DDEFEDFFAD530F5D8EEC2923274

: $00000001 F F$

In records, each two characters is a group to express a byte hexadecimal data except the starting mark ":", the second record is for example.

The first character is ":", it is a record start flag;

The 2 - 3 characters shown as "10" indicates the record contains $10 \mathrm{H}$ bytes code data;

The 4 - 7 characters shown as "0003" indicates that the start address of record codes is $0003 \mathrm{H}$;

The 8 - 9 characters shown as "00" indicates the record type is normal code data records;

The 10 - 41 characters is 16 bytes code data, hexadecimal codes are $02,00,1 \mathrm{~B}, 75,81,60, \mathrm{C} 2,92, \mathrm{D} 2, \mathrm{AF}, \mathrm{A} 2,93,40$, 04, D2, 88 and D2

The 42- 43 characters shown as "D2" indicates that the record checksum is D2;

The record end flags $0 \mathrm{DH}$ and $\mathrm{OAH}$ are not shown;

For text files, the data stored in document is each character ASCII, So, the data of the above record stored in file are $3 \mathrm{~A}$, $31,30,30,30,30,33,30,30,30,32,30,30,31,42,37,35,38$, $31,36,30,43,32,39,32,44,32,41,46,41,32,39,33,34,30$, $30,34,44,32,38,38,44,32,0 \mathrm{D}$ and $0 \mathrm{~A}$, they are total of 45 bytes.

In order to read the code in the code file, a CFile type object was defined, various operations on files was realized easily by calling member functions of the file object.

\section{2) The communication programming}

In order to simplify the program design, USB to UART chip is used so that the operation of the USB port is turned into a serial port operation. To implement serial communication, a Mscomm class object, $m$ _Comm, is defined. Through the $\mathrm{m}$ Comm member functions, it is easy to achieve the serial communication protocol settings as well as the serial port read and write

\section{CONCLUSION}

The single-chip microcomputer serial interface technology circuit board introduced in this paper is rich in function, advanced in technology and complete in document,it is used by many brothers colleges and universities and SCM technology training, and the good teaching effect has been achieved.

\section{ACKNOWLEDGMENTS}

We would like to thank teacher Jianguo Guo and Zhaoqing University for its support.

\section{REFERENCES}

[1] Maosheng Wu, Ximeng Wu. Design of the Single-chip Microcomputer Experiment Teaching System for Application.2011 International Conference on Electrical and Control Engineering (ICECE), V1.part II,p 649-652,2011.

[2] Wu Maosheng, Wu Ximeng, Liu Wenjuan. Application of the serial Port in Experiment Teaching and Development of embedded products. 2011 International Conference on Advanced Materials and Information Technology Processing (AMITP ), p 1884-1889, 2011.

[3] Atmel Corporatio. Atmel 8-bit Microcontroller with 4K Bytes In-System Programmable Flash AT89S51[EB/OL]. http://www.atmel.com/dyn/resources/prod_documents/doc2487.pdf, 2005, 3.

[4] Maosheng Wu, Jing Wang. A Study of in-System Programming Technology of the AT89S51 Microcontroller. 2012 Third International Conference on Mechanic Automation and Control Engineering. p 94-97, 2012 